\title{
Some Expressions of a Generalized Version of the Expected Time in the Red and the Expected Area in Red
}

\author{
JULIEN CALLANT, JULIEN TRUFIN \\ \& PIERRE ZUYDERHOFF \\ Department of Mathematics, Université Libre de Bruxelles (ULB) \\ Bruxelles, Belgium
}

\begin{abstract}
In this paper, we study a generalized version of the expected time in the red and the expected area in red introduced in Loisel (2005). We derive some expressions for this risk measure both in light tailed and heavy tailed cases for general risk processes. Then, some further expressions are obtained for Lévy processes together with an upper bound in the light-tailed case and an additional result for large initial capitals in the subexponential case.
\end{abstract}

Keywords: Expected time in the red; Expected area in red; Lévy processes; Upper bound; Asymptotic behavior. 


\section{Introduction}

Consider the surplus process (or risk process)

$$
U_{t}=u-X_{t}, \quad t \geq 0
$$

representing the surplus of an insurance company at time $t$ starting from some initial capital $U_{0}=u$, $X_{t}$ is the aggregate loss up to time $t$. For example, in the compound Poisson model, $X_{t}=S_{t}-c t$, where $c$ is the (constant) premium income per unit of time and $S_{t}$ is the total claim amount up to time $t$.

Dos Reis (1993) studies the total time below 0 for the surplus process, also called the total time in the red, defined as

$$
\tau(u)=\int_{t=0}^{\infty} 1_{\left\{U_{t}<0\right\}} d t
$$

where $1_{A}$ is the indicator function, as well as the expected total time below 0 given by

$$
\mathcal{T}(u)=E[\tau(u)]
$$

Loisel (2005) extends (2) and (3) by considering the time in the red until time $T \in[0, \infty]$, defined as

$$
\tau(u, T)=\int_{t=0}^{T} 1_{\left\{U_{t}<0\right\}} d t,
$$

and the expected time in the red until time $T$ given by

$$
\mathcal{T}(u, T)=E[\tau(u, T)]
$$

Loisel (2005) introduces the expected time-integrated negative part of the surplus process on $[0, T]$ for $T \in[0, \infty]$, also called the expected area in red. Formally, the expected area in red on $[0, T]$ is defined as

$$
\mathcal{A}(u, T)=E\left[\int_{t=0}^{T}\left|U_{t}\right| 1_{\left\{U_{t}<0\right\}} d t\right] .
$$

In particular, Loisel (2005) states and proves differentiation theorems for $\mathcal{A}(u, T)$ linking $\mathcal{A}(u, T)$ to the expected time in the red $\mathcal{T}(u, T)$. Biard et al. (2010) investigate the asymptotic behavior of $\mathcal{A}(u, T)$ as $u \rightarrow \infty$ and Loisel and Trufin (2014) study the smallest initial capital needed to ensure that the expected area in red is less than a given predetermined value. Macci (2008) proves that $\mathcal{A}(u, T)$ and $\mathcal{T}(u, T)$ satisfy a large deviation principle for Lévy processes and gives explicit expression of the rate function.

As noticed in Loisel (2005), both $\mathcal{A}(u, T)$ and $\mathcal{T}(u, T)$ can be seen as particular cases of

$$
\mathcal{E}_{g}(u, T)=E\left[\int_{0}^{T} e^{-\rho t} g\left(U_{t}\right) d t\right]
$$

where $g: \mathbb{R} \rightarrow \mathbb{R}^{+}$is a locally integrable function with a negative support and $\rho$ is a nonnegative 
constant. When $\rho=0$, expression (7) with $g(x)=1_{\{x<0\}}$ and $g(x)=|x| 1_{\{x<0\}}$ gives $\mathcal{T}(u, T)$ and $\mathcal{A}(u, T)$, respectively.

In this paper, we study $\mathcal{E}_{g}(u, T)$ for functions $g$ assumed to be positive with a negative support and locally integrable under mild assumptions given in Section 2. The approach adopted in this paper to study $\mathcal{E}_{g}(u, T)$ relies on the notion of generalized function (or distribution) initially formalized in Schwartz (1945). To our knowledge, Schwartz's theory of distributions has not yet been used in the context of ruin theory. This new angle to tackle ruin problems enables us to obtain results in terms of derivatives (as in Proposition 3) and Fourier transforms (the results in Section 3). In particular, we get results generalizing the ones of Loisel (2005).

This paper is structured as follows. In Section 2, we prove that the generalized version of the expected time in the red and the expected area in red defined in $(7)$, namely $\mathcal{E}_{g}(u, T)$ can be written as the convolution of $g$ and

$$
f: u \in \mathbb{R} \rightarrow \int_{0}^{T} e^{-\rho t} f_{X_{t}}(u) d t
$$

where $f_{X_{t}}(u) d u=d F_{X_{t}}(u)$. Then, we rely on the differentiation theorems of Loisel (2005) to show that, when $\rho=0$, if $\left\{S_{t}, t \geq 0\right\}$ is a jump process having a finite expected number of nonnegative jumps in every finite time interval and if the distribution function of $S_{t}$ has no atom on $(0, \infty)$ for all $t \geq 0$, then we have

$$
f(u)=\frac{1}{c} E\left[N^{0}(u, T)\right]
$$

where $N^{0}(u, T)$ is the number of times the surplus process $\left\{U_{t}, t \geq 0\right\}$ takes the value 0 on the time interval $[0, T]$, that is,

$$
N^{0}(u, T)=\operatorname{card}\left(\left\{t \in[0, T], U_{t}=0\right\}\right) .
$$

In Section 3, considering Lévy processes, we derive an analytical expression for $f$ and we establish expressions for $\mathcal{E}_{g}(u, T)$ in the particular cases where $g(x)=|x|^{n} 1_{\{x<0\}}, n \in \mathbb{N}$. These expressions can be used to perform efficient numerical computations, as illustrated in the compound Poisson case with exponential claim sizes where explicit expressions (obtained in Loisel (2005) by a different approach) for $\mathcal{T}(u, \infty)$ and $\mathcal{A}(u, \infty)$ are available. In particular, one sees that our results tend to $\mathcal{T}(u, \infty)$ and $\mathcal{A}(u, \infty)$ when $T \rightarrow \infty$, as expected. Next, in Section 4 , an upper bound is also obtained on $\mathcal{E}_{g}(u, T)$ for light-tailed claims. Finally, in Section 5, we characterize the asymptotic behavior of $\mathcal{E}_{g}(u, T)$ as $u \rightarrow \infty$ for subexponential claim distributions.

\section{$2 \quad$ Expression for $\mathcal{E}_{g}(u, T)$}

In order to define $f$ in a more rigorous way than (8), we recall the concept of generalized functions. A generalized function is a continuous linear functional on the set $\mathcal{K}$ of all real functions $\phi$ with bounded support and with continuous derivatives of all orders, referred to as test functions. The real number associated to a generalized function $\tilde{f}$ and a test function $\phi$ is denoted $(\tilde{f}, \phi)$. If for any test function $\phi$, there exists a locally integrable function, denoted $\hat{f}(u)$ such that

$$
(\tilde{f}, \phi)=\int_{-\infty}^{\infty} \hat{f}(u) \phi(u) d u
$$


then $\tilde{f}$ is called regular. We assume that the generalized function

$$
f: \phi \in \mathcal{K} \rightarrow(f, \phi)=\int_{0}^{T}\left(\int_{-\infty}^{\infty} \phi(x) d F_{X_{t}}(x)\right) e^{-\rho t} d t
$$

is regular. This is a mild assumption and the results of this section hold for very general processes. For example, in case of a constant process, $f_{X_{t}}=\delta, \forall t \in[0, T]$, where $\delta$ denotes the Dirac delta function, and $f$ is not regular in 0 while when $X_{t}=S_{t}-c t$ with $c \in \mathbb{R}$ and $S_{t}$ is a Brownian motion, $f$ is regular. Henceforth, we denote a regular generalized function and its associated locally integrable function with the same symbol. Namely, in the rest of the paper, we write

$$
(f, \phi)=\int_{-\infty}^{\infty} f(x) \phi(x) d x .
$$

In this section and in Section 3, we use the derivative in the sense of generalized function theory, which enables us to consider the derivative of piecewise differentiable functions, such as the Heaviside function $\theta$, that is

$$
\theta^{\prime}(x)=\delta(x)
$$

The derivative $D \tilde{f}$ of a generalized function $\tilde{f}$ is defined as

$$
(D \tilde{f}, \phi)=\left(\tilde{f},-\phi^{\prime}\right),
$$

where $\phi^{\prime}$ is the derivative of a test function $\phi$. Note that $f$ is equivalently defined as

$$
f=\int_{0}^{T} e^{-\rho t}\left(D F_{X_{t}}\right) d t
$$

We refer the interested reader to Gel'fand and Shilov (1964) for more details about the generalized functions.

First, we show that $\mathcal{E}_{g}(u, T)$ can be written as the convolution of $g$ and $f$ defined in (8).

Proposition 1 We have

$$
\mathcal{E}_{g}(u, T)=(f * g)(u)=\int_{-\infty}^{\infty} f(y) g(u-y) d y, \quad u \in \mathbb{R} .
$$

Proof. We have

$$
\begin{aligned}
\mathcal{E}_{g}(u, T) & =\int_{0}^{T} E\left[e^{-\rho t} g\left(U_{t}\right)\right] d t \\
& =\int_{0}^{T} e^{-\rho t}\left(\int_{-\infty}^{\infty} g(u-y) d F_{X_{t}}(y)\right) d t \\
& =\int_{0}^{T} e^{-\rho t}\left(\int_{-\infty}^{\infty} f_{X_{t}}(y) g(u-y) d y\right) d t
\end{aligned}
$$


where $f_{X_{t}}(y) d y=d F_{X_{t}}(y)$. Applying Fubini's theorem, we get

$$
\mathcal{E}_{g}(u, T)=\int_{-\infty}^{\infty}\left(\int_{0}^{T} e^{-\rho t} f_{X_{t}}(y) d t\right) g(u-y) d y .
$$

The latter proposition directly leads to the following result.

Corollary 2 If we further assume that $g$ is a continuously differentiable function on $(-\infty, 0)$, then we have

$$
\frac{d \mathcal{E}_{g}(u, T)}{d u}=\mathcal{E}_{g^{\prime}}(u, T)-g\left(0^{-}\right) f(u),
$$

for almost every $u \in \mathbb{R}$ and where $g^{\prime}$ denotes the almost everywhere derivative of $g$.

Proof. The derivative of (7) in the sense of distribution gives

$$
D(f * g)=f * D g=\left(f * g^{\prime}\right)-\left(f * g\left(0^{-}\right) \delta\right)=\left(f * g^{\prime}\right)-g\left(0^{-}\right) f,
$$

where $g^{\prime}$ denotes the almost everywhere derivative of $g$. Now, the correspondence between regular distributions and locally integrable functions preserves the operations of addition, multiplication by a number and differentiation, so that we get (10).

Based on Theorem 3 in Loisel (2005), one can prove that $f(u)=\frac{1}{c} E\left[N^{0}(u, T)\right]$ when $\rho=0$ and $S_{t}$ is a jump process satisfying hypothesis H1 in Loisel (2005), as shown next.

Proposition 3 Assume that $X_{t}=S_{t}$-ct where $S_{t}$ is a spectrally positive jump process with a finite expected number of jumps in every finite interval and assume that, for each $t$, the distribution of $S_{t}$ has no atom on $(0, \infty)$. Then, when $\rho=0$, we have

$$
f(u)=\frac{1}{c} E\left[N^{0}(u, T)\right]
$$

Proof. It is clear that

$$
f(u)=\int_{0}^{T} f_{S_{t}-c t}(u) d t=\int_{0}^{T} f_{S_{t}-c t \mid S_{t}>0}(u) P\left[S_{t}>0\right] d t+\int_{0}^{T} \delta(c t+u) P\left[S_{t}=0\right] d t
$$

is continuous at $u \in \mathbb{R} \backslash\{0,-c T\}$ when $S_{t}$ has no atom on $(0, \infty)$. We apply Corollary 2 with $g(x)=1_{x<0}$ and we get

$$
\frac{d \mathcal{T}(u, T)}{d u}=-f(u)
$$

for $u \in \mathbb{R} \backslash\{0,-c T\}$. Then, it suffices to notice that Theorem 3 in Loisel (2005) can be extended to $u \in \mathbb{R} \backslash\{0,-c T\}$ so that we get

$$
f(u)=-\frac{d}{d u} \mathcal{T}(u, T)=\frac{1}{c} E\left[N^{0}(u, T)\right], \quad u \in \mathbb{R} \backslash\{0,-c T\} .
$$


Note that the proof of this extension is similar to the one of Theorem 3 in Loisel (2005) for $-c T<u<0$ and is obvious for $u<-c T$. Finally, since

$$
\begin{aligned}
f(0) & =\int_{[0, T]} f_{S_{t}}(c t) d t=\frac{1}{c} P\left[S_{0}=0\right]+\int_{] 0, T]} f_{S_{t}}(c t) d t \\
& =\frac{1}{c}+\frac{1}{c} E\left[N^{0}\left(0^{+}, T\right)\right]=\frac{1}{c} E\left[N^{0}(0, T)\right]
\end{aligned}
$$

and

$$
f(-c T)=\int_{0}^{T} \delta(c t-c T) P\left[S_{t}=0\right] d t=\frac{1}{c} P\left[S_{T}=0\right]=\frac{1}{c} E\left[N^{0}(-c t, T)\right],
$$

the announced result is directly obtained from Equations (11), (12) and (13).

Remark 4 As mentioned in Loisel (2005), when $\rho=0, f(u)$ corresponds to the expectation of

$$
L_{0}(u, T)=\lim _{\epsilon \rightarrow 0}\left(\frac{1}{2 \epsilon} \int_{0}^{T} 1_{\left\{\left|U_{t}\right|<\epsilon\right\}} d t\right)
$$

which is the local time spent at 0 by the process $\left\{U_{t}, t \geq 0\right\}$ up to time $T$. Notice that $f(u+y)$ is the expectation of the local time spent at the level $-y$ by the process $\left\{U_{t}, t \geq 0\right\}$ up to time $T$, i.e.

$$
f(u+y)=E\left[L_{-y}(u, T)\right],
$$

so that the expectation of the time spent between two levels $a$ and $b$ (with $a>b>0$ ) by the process $\left\{U_{t}, t \geq 0\right\}$ up to time $T$ is given by

$$
\int_{-a}^{-b} f(u+y) d y
$$

which generalizes the notion of expected time in the red.

The support of $g$ is bounded from the right by zero. Hence, Equation (9) can be rewritten as

$$
\mathcal{E}_{g}(u, T)=\int_{u}^{\infty} f(y) g(u-y) d y, \quad u \in \mathbb{R} .
$$

Notice that under the assumptions of Proposition 3 the support of $f$ is bounded from the left by $-c T$. Moreover, for $u>0$, we have

$$
f(u) \leq \frac{E\left[N^{0}(u, T)\right]}{c} \leq \frac{E\left[N_{T}\right]}{c} \psi(u, T) \leq \frac{E\left[N_{T}\right]}{c} P\left[S_{T}>u\right]
$$

where $N_{T}$ is the number of jumps for $S_{t}$ up to time $T$ and $\psi(u, T)$ is the finite time ruin probability, so that the integral in (14) converges when the function $u \rightarrow \psi(u, T) g(-u)$ decays faster than $1 / u$. 


\section{Lévy process}

Let us denote

$$
\kappa(z)=\log \left(E\left[e^{z X_{1}}\right]\right)
$$

for $z \in \mathbb{C}$. Henceforth, we assume that $\left\{X_{t}, t \geq 0\right\}$ is a Lévy process. Hence, $\log \left(E\left[e^{z X_{t}}\right]\right)=t \kappa(z)$ and $\kappa(z)$ has the Lévy-Khinchine representation

$$
\kappa(z)=a z-\frac{\sigma^{2} z^{2}}{2}+\int_{-\infty}^{\infty}\left(e^{z x}-1-z x 1_{|x| \leq 1}\right) \nu(d x),
$$

where $a \in \mathbb{R}, \sigma \geq 0$ and $\nu$ is the Lévy measure satisfying the property

$$
\int_{\mathbb{R}}\left(x^{2} \wedge 1\right) \nu(d x)<\infty .
$$

One refers to $\left(a, \sigma^{2}, \nu\right)$ as the characteristic triplet of $X_{t}$. Notice that the net profit condition $E\left[X_{1}\right]<0$ is not required in this section unless explicitly mentioned.

\subsection{Fourier transform}

We denote by $L^{p}(\mathbb{R}), p=1,2$, the space of all measurable functions $\tilde{f}: \mathbb{R} \rightarrow \mathbb{C}$ such that

$$
\int_{-\infty}^{\infty}|\tilde{f}(x)|^{p} d x<\infty
$$

The Fourier transform of a function $\tilde{f} \in L^{1}(\mathbb{R})$ is defined as

$$
\mathcal{F}_{u}[\tilde{f}(u)](\omega)=\int_{-\infty}^{\infty} e^{i \omega u} \tilde{f}(u) d u .
$$

For a function $\tilde{f} \in L^{2}(\mathbb{R})$, expression (15) does not necessarily exist. However, the Fourier transform (15) can be extended from $L^{1}(\mathbb{R}) \cap L^{2}(\mathbb{R})$ to $L^{2}(\mathbb{R})$ by using $\lim _{s \rightarrow \infty} \int_{-s}^{s} e^{i \omega u} \tilde{f}(u) d u$. In the following, the Fourier transform of a function $\tilde{f} \in L^{2}(\mathbb{R})$ is also denoted by $\mathcal{F}_{u}[\tilde{f}(u)](\omega)$. The Fourier transform $\mathcal{F}_{u}[\tilde{f}(u)](\omega)$ is an automorphism of $L^{2}(\mathbb{R})$, so that it is invertible. We denote its inverse by $\mathcal{F}_{\omega}^{-1}[\tilde{f}(\omega)](u)$ and we get

$$
\mathcal{F}_{\omega}^{-1}[\tilde{f}(\omega)](u)=\frac{1}{2 \pi} \lim _{s \rightarrow \infty} \int_{-s}^{s} e^{-i \omega u} \tilde{f}(u) d u \text {. }
$$

The Fourier transform can be extended to the class of locally integrable functions and more widely to generalized functions (see e.g. Gel'fand and Shilov (1964)). The Fourier transform $\mathcal{F}[f]$ of a generalized function $f$ is defined as

$$
(\mathcal{F}[f], \mathcal{F}[\phi])=2 \pi(f, \phi),
$$


where $\mathcal{F}[\phi]$ is the Fourier transform of the test function $\phi$. In the following, we use the same notation for Fourier transforms of generalized functions than for $L^{1}(\mathbb{R})$ and $L^{2}(\mathbb{R})$ functions. Note that the Fourier transform of a locally integrable function which, as $|x| \rightarrow \infty$, increases no faster than some power of $|x|$ has a Fourier transform in the sense of generalized functions given by

$$
\lim _{s \rightarrow \infty} \int_{-s}^{s} e^{i \omega u} \tilde{f}(u) d u
$$

where the the symbol lim holds for the weak limit. One says that a sequence of generalized functions $\left\{f_{n}\right\}_{n}$ converges weakly to $f$ if $\left(f_{n}, \phi\right) \rightarrow(f, \phi)$, for all test function $\phi$. In the sequel, all the limits have to be understood as weak limits.

\subsection{Expression for $f$}

The next result gives an expression of $\mathcal{F}_{u}[f(u)](\omega)$ almost everywhere.

\section{Lemma 5}

$$
\mathcal{F}_{u}[f(u)](\omega)=\frac{e^{T(\kappa(i \omega)-\rho)}-1}{\kappa(i \omega)-\rho} .
$$

Proof. We first show that $f$ is integrable when $T<\infty$ since

$$
\int_{-\infty}^{\infty} f(u) d u=\int_{-\infty}^{\infty}\left(\int_{0}^{T} f_{X_{t}}(u) d t\right) d u=\int_{0}^{T}\left(\int_{-\infty}^{\infty} d F_{X_{t}}(u)\right) d t=T<\infty
$$

The Fourier transform of $f$ is then given by

$$
\begin{aligned}
\mathcal{F}_{u}[f(u)](\omega) & =\int_{-\infty}^{\infty} e^{i \omega u} f(u) d u \\
& =\int_{-\infty}^{\infty} e^{i \omega u}\left(\int_{0}^{T} e^{-\rho t} f_{X_{t}}(u) d t\right) d u \\
& =\int_{0}^{T} e^{-\rho t} E\left[e^{i \omega X_{t}}\right] d t \\
& =\int_{0}^{T} e^{t(\kappa(i \omega)-\rho)} d t \\
& = \begin{cases}\frac{e^{T(\kappa(i \omega)-\rho)}-1}{\kappa(i \omega)-\rho} & \text { for } \kappa(i \omega)-\rho \neq 0, \\
T & \text { for } \kappa(i \omega)-\rho=0 .\end{cases}
\end{aligned}
$$

Applying the inverse Fourier transform, we are now able to derive the following expression for $f$. 
Proposition 6 We have

$$
f(u)=\frac{1}{\pi} \int_{0}^{\infty} \mathfrak{R e}\left(\frac{e^{T(\kappa(i \omega)-\rho)}-1}{\kappa(i \omega)-\rho} e^{-i u \omega}\right) d \omega
$$

almost everywhere, where $\mathfrak{R e}(z)$ denotes the real part of the complex number $z$.

Proof. Notice that

$$
\frac{e^{T(\kappa(-i \omega)-\rho)}-1}{\kappa(-i \omega)-\rho} e^{i u \omega}=\left(\frac{e^{T(\kappa(i \omega)-\rho)}-1}{\kappa(i \omega)-\rho} e^{-i u \omega}\right)^{*},
$$

where we denote by $z^{*}$ the complex conjugate of the complex number $z$. Hence, the announced result follows from the application of the inverse Fourier transform to (16), i.e.

$$
\begin{aligned}
f(u) & =\mathcal{F}_{\omega}^{-1}\left[\frac{e^{T(\kappa(i \omega)-\rho)}-1}{\kappa(i \omega)-\rho}\right](u)=\frac{1}{2 \pi} \lim _{s \rightarrow \infty} \int_{-s}^{s} \frac{e^{T(\kappa(i \omega)-\rho)}-1}{\kappa(i \omega)-\rho} e^{-i u \omega} d \omega \\
& =\frac{1}{2 \pi} \int_{0}^{\infty}\left(\frac{e^{T(\kappa(i \omega)-\rho)}-1}{\kappa(i \omega)-\rho} e^{-i u \omega}+\frac{e^{T(\kappa(-i \omega)-\rho)}-1}{\kappa(-i \omega)-\rho} e^{i u \omega}\right) d \omega .
\end{aligned}
$$

Of course, inserting (17) into (9) enables to compute $\mathcal{E}_{g}(u, T)$ for $T<\infty$.

We now consider the case when $T$ tends to infinity.

Proposition 7 Let $S_{t}$ be a spectrally positive Lévy process such that $E\left[X_{1}\right]<0$ and such that the distribution of $S_{1}-c$ is not lattice. Then, when $\rho=0$, we have

$$
f(u ; \infty)=\lim _{T \rightarrow \infty} f(u ; T)=\frac{\psi(u)}{\left|E\left[X_{1}\right]\right|}
$$

for almost all $u \in \mathbb{R}$, where we made explicit the dependency in $T$ for the function $f$.

Proof. First notice that

$$
\lim _{T \rightarrow \infty} \frac{e^{i \omega T}-1}{i \omega}=-\frac{1}{i \omega}+\pi \delta(\omega) .
$$

Letting $T$ tends to infinity in (16) when $\rho=0$ gives

$$
\begin{aligned}
\mathcal{F}_{u}[f(u ; \infty)](\omega) & =\lim _{T \rightarrow \infty} \mathcal{F}_{u}[f(u ; T)](\omega) \\
& =-\frac{1}{\kappa(i \omega)}+\pi \delta(-i \kappa(i \omega)) \\
& =-\frac{1}{\kappa(i \omega)}+\frac{\pi \delta(\omega)}{\kappa^{\prime}(0)} \\
& =-\frac{1}{\kappa(i \omega)}+\frac{\pi \delta(\omega)}{\left|E\left[X_{1}\right]\right|},
\end{aligned}
$$


where the interchanging of the limit and the Fourier transform is justified by Fubini's theorem and where the third equality is obtained thanks to the assumption that the distribution of $S_{1}-c$ is not lattice. Theorem XI.2.4 of Albrecher and Asmussen (2010) gives the Laplace transform of $\psi(u)$ when $E\left[X_{1}\right]<0$ and when the jump part of $S_{t}$ is spectrally positive, so that the Fourier transform of $\psi(u)$ is given by

$$
\begin{aligned}
\mathcal{F}_{u}[\psi(u)](\omega) & =\mathcal{F}_{u}\left[1_{u<0}\right](\omega)+\mathcal{F}_{u}\left[\psi(u) 1_{u \geq 0}\right](\omega) \\
& =\left(\frac{1}{i \omega}+\pi \delta(\omega)\right)+\left(-\frac{1}{i \omega}+\frac{E\left[X_{1}\right]}{\kappa(i \omega)}\right) \\
& =-\frac{\left|E\left[X_{1}\right]\right|}{\kappa(i \omega)}+\pi \delta(\omega) .
\end{aligned}
$$

The result is then obtained by comparing the Fourier transforms (19) and (20).

Note that when $S_{t}$ is a compound Poisson process, Expression (18) can be rewritten as

$$
f(u ; \infty)=\frac{1}{c} E\left[N^{0}(u, \infty)\right]=\frac{\psi(u)}{c(1-\psi(0))} .
$$

In this particular case, Expression (21) is obtained with a different approach in Theorem 6 of Loisel (2005). It is worth mentioning that this result can also be derived by considering the limit when $T$ tends to infinity of both sides of the standard Seal formula, i.e.,

$$
\psi(u, t)=P\left[S_{T}-c T>u\right]+\int_{0}^{T}(1-\psi(0, T-t)) f_{S_{t}-c t}(u) c d t .
$$

The next result is a direct consequence of (9) and (18) and gives an expression for $\mathbb{E}_{g}(u, T)$ when $T=\infty$.

Corollary 8 Let $S_{t}$ be a spectrally positive Lévy process such that $E\left[X_{1}\right]<0$. When $\rho=0$,

$$
\mathcal{E}_{g}(u, \infty)=\frac{(\psi * g)(u)}{\left|E\left[X_{1}\right]\right|}
$$

for almost all $u \in \mathbb{R}$.

\subsection{Expression for $\mathcal{E}_{g}(u, T)$ when $g(x)=|x|^{n} 1_{\{x<0\}}, n \in \mathbb{N}$}

The cases $g(x)=|x|^{n} 1_{\{x<0\}}, n \in \mathbb{N}$, are interesting ones. When $\rho=0$, recall that for $n=0$, $\mathcal{E}_{g}(u, T)$ is the expected time in the red while for $n=1, \mathcal{E}_{g}(u, T)$ is the expected area in red. In these cases, an expression for $\mathcal{E}_{g}(u, T)$ can directly be obtained by considering the Fourier transform of (9), as shown next. 
Proposition 9 If $g(x)=|x|^{n} 1_{\{x<0\}}, n \in \mathbb{N}$, then

$$
\mathcal{E}_{g}(u, T)=\mathcal{F}_{\omega}^{-1}\left[\left((-i)^{n+1} n ! \omega^{-n-1}+i^{n} \pi \delta^{(n)}(\omega)\right) \frac{e^{T(\kappa(i \omega)-\rho)}-1}{\kappa(i \omega)-\rho}\right](u), \quad u>0,
$$

where $\delta^{(n)}$ is the $n^{\text {th }}$ derivative of the Dirac delta function.

Proof. The Fourier transform of the generalized function $|x|^{n} 1_{\{x<0\}}$ is given by

$$
\mathcal{F}_{x}\left[|x|^{n} 1_{\{x<0\}}\right](\omega)=(-i)^{n+1} n ! \omega^{-n-1}+i^{n} \pi \delta^{(n)}(\omega) .
$$

See for instance Gel'fand and Shilov (1964). Hence, by (16), a simple application of the convolution theorem to Proposition 1 directly leads to (23).

In particular, we get the following results for $n=0$ and $n=1$.

\section{Corollary 10 We have}

$$
\mathcal{T}(u, T)=\frac{1}{\pi} \int_{0}^{\infty} \frac{1}{\omega} \mathfrak{I m}\left(\frac{e^{T \kappa(i \omega)}-1}{\kappa(i \omega)} e^{-i \omega u}\right) d \omega+\frac{1}{2} T, \quad u>0,
$$

where $\mathfrak{I m}(z)$ denotes the imaginary part of the complex number $z$.

Proof. For $n=0$, it suffices to notice that Equation (23) simplifies to

$$
\begin{aligned}
\mathcal{E}_{g}(u, T) & =\mathcal{F}_{\omega}^{-1}\left[-\frac{i}{\omega} \frac{e^{T \kappa(i \omega)}-1}{\kappa(i \omega)}\right](u)+\mathcal{F}_{\omega}^{-1}\left[\pi \delta(\omega) \frac{e^{T \kappa(i \omega)}-1}{\kappa(i \omega)}\right](u) \\
& =\frac{1}{2 \pi} \int_{0}^{\infty}-\frac{i}{\omega}\left(\frac{e^{T \kappa(-i \omega)}-1}{\kappa(-i \omega)} e^{-i \omega u}-\frac{e^{T \kappa(i \omega))}-1}{\kappa(i \omega)} e^{i \omega u}\right) d \omega+\frac{1}{2} T .
\end{aligned}
$$

Notice that

$$
\mathcal{T}(u, T)=\mathcal{F}_{\omega}^{-1}\left[-\frac{i}{\omega} \frac{e^{T \kappa(i \omega)}-1}{\kappa(i \omega)}\right](u)+\frac{1}{2} T=-\frac{1}{2} \int_{-\infty}^{\infty} f(v) \operatorname{sgn}(u-v) d v+\frac{1}{2} T,
$$

where

$$
\operatorname{sgn}(x)=\left\{\begin{array}{cl}
-1 & \text { for } x<0 \\
1 & \text { for } x \geq 0
\end{array}\right.
$$

so that $\lim _{u \rightarrow-\infty} \mathcal{T}(u, T)=\frac{1}{2} \int_{\infty}^{\infty} d v f(v)+\frac{1}{2} T=T$ and $\lim _{u \rightarrow+\infty} \mathcal{T}(u, T)-\frac{1}{2} \int_{\infty}^{\infty} d v f(v)+\frac{1}{2} T=0$, as expected. 
Corollary 11 When $g(x)=|x| 1_{\{x<0\}}$, we have

$$
\mathcal{E}_{g}(u, T)=-\frac{1}{\pi} \int_{0}^{\infty} \frac{1}{\omega^{2}}\left(\mathfrak{R e}\left(\frac{e^{T(\kappa(i \omega)-\rho)}-1}{\kappa(i \omega)-\rho} e^{-i \omega u}\right)-T\right) d \omega-\frac{1}{2} u T-\frac{T^{2}}{4}(c-\lambda \mu), \quad u>0 .
$$

In particular, we get

$$
\mathcal{A}(u, T)=-\frac{1}{\pi} \int_{0}^{\infty} \frac{1}{\omega^{2}}\left(\mathfrak{R e}\left(\frac{e^{T \kappa(i \omega)}-1}{\kappa(i \omega)} e^{-i \omega u}\right)-T\right) d \omega-\frac{1}{2} u T-\frac{T^{2}}{4}(c-\lambda \mu), \quad u>0 .
$$

Proof. For $n=1$, Equation (23) simplifies to

$$
\begin{aligned}
\mathcal{E}_{g}(u, T)= & \mathcal{F}_{\omega}^{-1}\left[-\frac{1}{\omega^{2}} \frac{e^{T(\kappa(i \omega)-\rho)}-1}{\kappa(i \omega)-\rho}\right](u)+\mathcal{F}_{\omega}^{-1}\left[i \pi \delta^{\prime}(\omega) \frac{e^{T(\kappa(i \omega)-\rho)}-1}{\kappa(i \omega)-\rho}\right](u) \\
= & \frac{1}{2 \pi} \int_{0}^{\infty}-\frac{1}{\omega^{2}}\left(\frac{e^{T(\kappa(i \omega)-\rho)}-1}{\kappa(i \omega)-\rho} e^{-i \omega u}+\frac{e^{T \kappa(-i \omega)-\rho}-1}{\kappa(-i \omega)-\rho} e^{i \omega u}-2 T\right) d \omega \\
& \quad-\frac{1}{2} u T-\frac{T^{2}}{4}(c-\lambda \mu),
\end{aligned}
$$

which is the announced result for $\mathcal{E}_{g}(u, T)$. The expression (26) directly follows from (25) with $\rho=0$.

Notice that expressions (17) and (24) can be obtained from expressions (24) and (26), respectively, by using Corollary 2.

\subsection{Numerical illustration}

In this section, we assume that $X_{t}=S_{t}-c t$ with $\left\{S_{t}, t \geq 0\right\}$ a compound Poisson process. That is, we suppose that

$$
S_{t}=\sum_{k=1}^{N_{t}} W_{k}, \quad t \geq 0
$$

where $\left\{N_{t}, t \geq 0\right\}$ is a Poisson process of rate $\lambda>0$ and claim sizes $\left\{W_{k}, k \geq 1\right\}$ are assumed to be independent and identically distributed as $W \geq 0$ with mean $\mu<\infty$ and distribution function $F_{W}$. The claim sizes $\left\{W_{k}, k \geq 1\right\}$ are also assumed to be independent of $\left\{N_{t}, t \geq 0\right\}$. Notice that, in this case, the charasteristic exponent $\kappa(z)$ is given by

$$
\kappa(z)=\log \left(E\left[e^{z\left(S_{1}-c\right)}\right]\right)=\lambda(\xi(z)-1)-c z
$$

for $z \in \mathbb{C}$ with $\xi(z)=E\left[e^{z W}\right]$.

Figures 1 and 3 display numerical evaluations with Wolfram Mathematica of Expressions (24) and (26) for different values of $u$ and $c$ when $W$ is exponentially distributed. In the exponential case, explicit expressions for $\mathcal{T}(u, \infty)$ and $\mathcal{A}(u, \infty)$ can be found in Loisel (2005). We notice that 

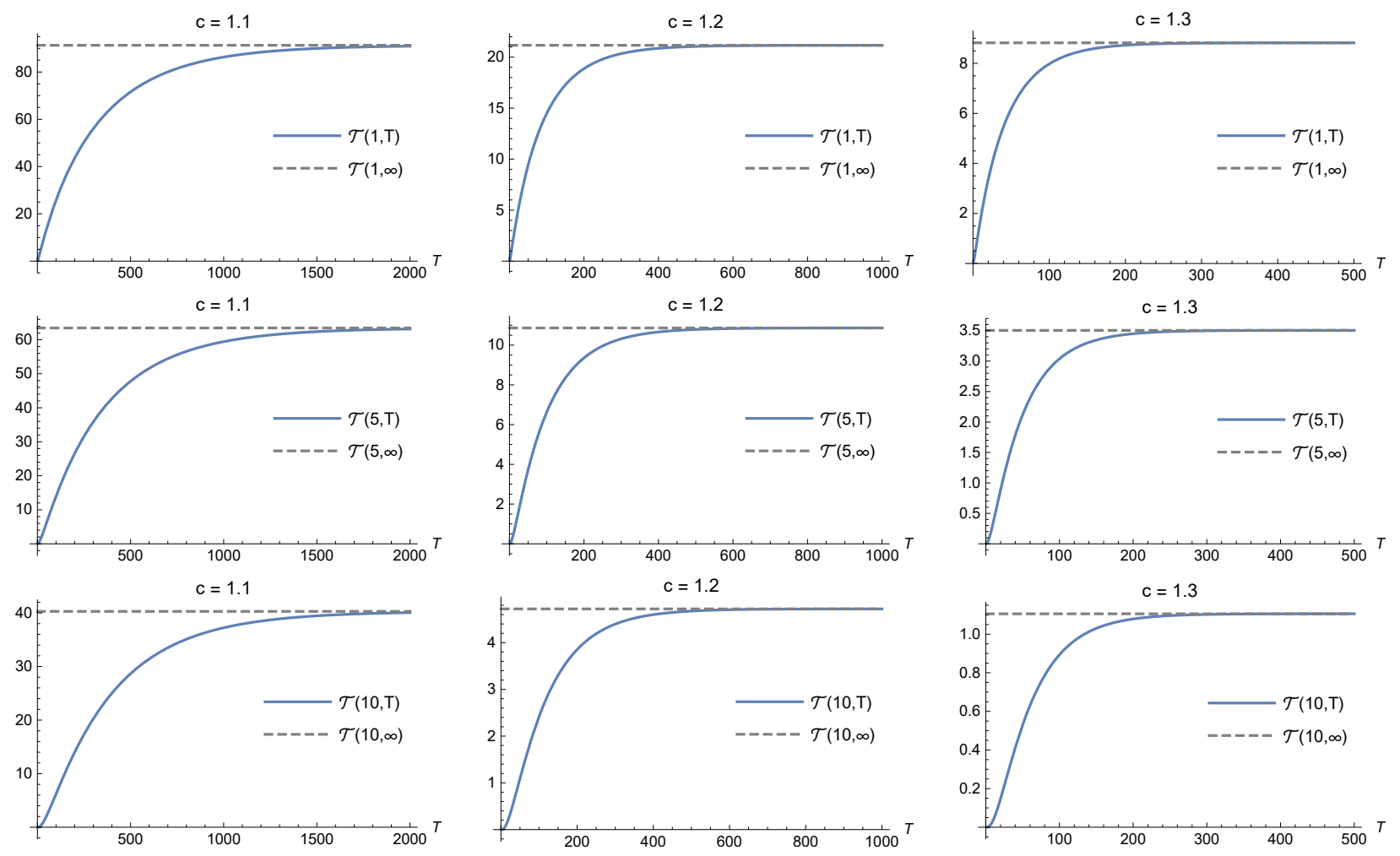

Figure 1: Numerical evaluation of $\mathcal{T}(u, T)$ for different values of $c$ and $u$ when $\lambda=1$ and $W \sim$ $\operatorname{Exp}(1)$.

our results tend to $\mathcal{T}(u, \infty)$ and $\mathcal{A}(u, \infty)$ when $T$ becomes large, as expected. Note that in the evaluation of $\mathcal{T}(u, T)$, the integrand of $(24)$ is highly oscillating. We approximate this expression by taking a finite upper bound $x$ in the integral. The approximation error is easily proven to be $\mathcal{O}(1 / x)$ so that it can be taken as small as wanted. Figure 2 illustrates that the value of the approximation as a function of $x$ converges quickly for specific values of $u$ and $c$. For $\mathcal{A}(u, T)$, the integrand in (26) is not oscillating (due to the term $-T$ ) and the integral converges more quickly.

\section{Upper bound for light tailed claim distributions}

In this section, we assume that $F_{X_{1}}$ is a light tailed distribution. Furthermore, we suppose that the safety loading condition $E\left[X_{1}\right]<0$ is fulfilled. Then, as shown in the next result, an upper bound for $\mathcal{E}_{g}(u, T)$ can be derived when $g$ satisfies

$$
g(x) \leq K e^{-p x}
$$

for all $x \in \mathbb{R}$, where $K$ is a positive constant.

Proposition 12 Let $p$ be a strictly positive constant such that $E\left[e^{p X_{1}}\right]$ exists and $g$ a real function 


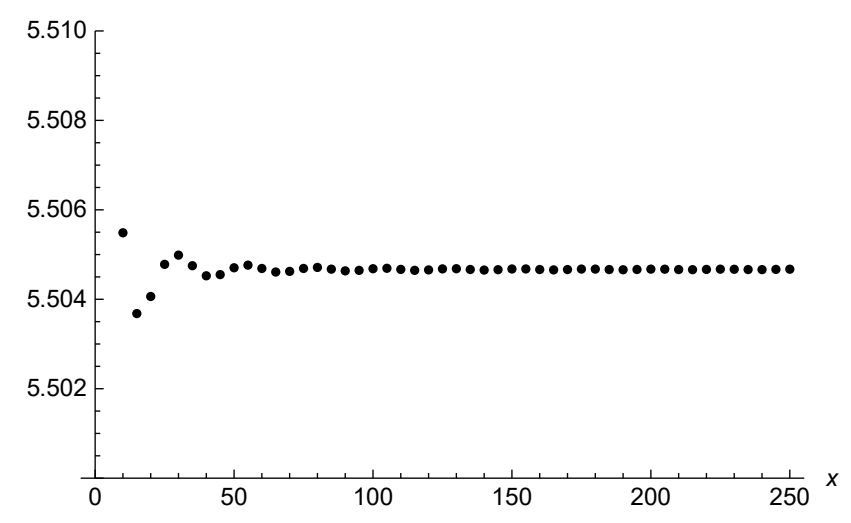

Figure 2: Convergence of the approximation $\frac{1}{\pi} \int_{0}^{x} \frac{1}{\omega} \mathfrak{I m}\left(\frac{e^{T \kappa(i \omega)}-1}{\kappa(i \omega)} e^{-i \omega u}\right) d \omega+\frac{1}{2} T$ with respect to $x$, when $u=1, T=20, \lambda=1, c=1.1$ and $W \sim \operatorname{Exp}(1)$.
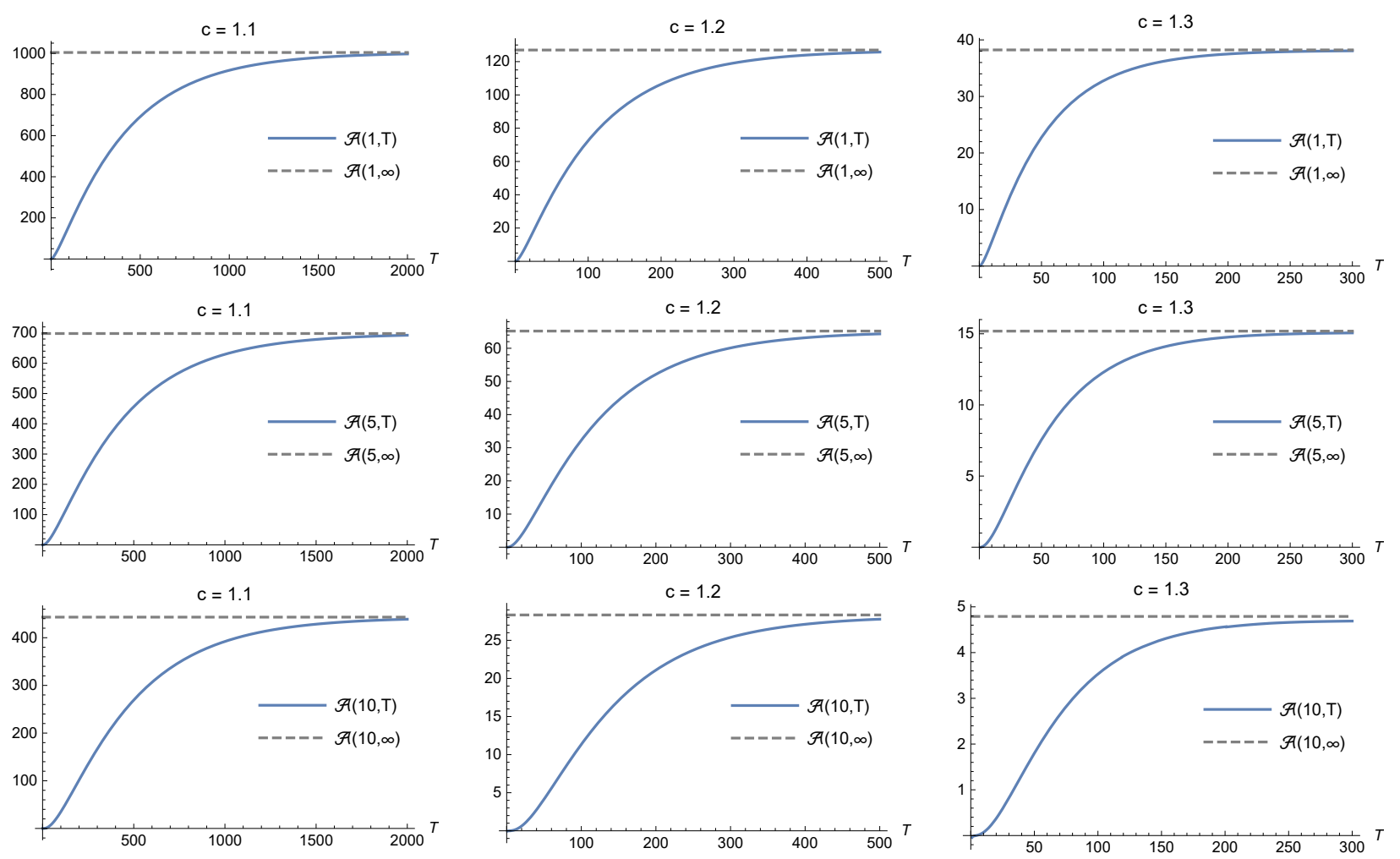

Figure 3: Numerical evaluation of $\mathcal{A}(u, T)$ for different values of $c$ and $u$ when $\lambda=1$ and $W \sim$ $\operatorname{Exp}(1)$. 
with negative support satisfying (28). Then, for all $u \geq 0$, we get

$$
\mathcal{E}_{g}(u, T) \leq\left\{\begin{array}{ll}
K \frac{e^{T(\kappa(p)-\rho)}-1}{\kappa(p)-\rho} e^{-p u}, & \text { if } \kappa(p)-\rho \neq 0 \\
K T e^{-p u}, & \text { if } \kappa(p)-\rho=0
\end{array} .\right.
$$

Proof. For Lévy processes, we have

$$
e^{-\rho t} E\left[e^{-p U_{t}}\right]=e^{-p u} e^{t(\kappa(p)-\rho)} .
$$

Therefore, we get

$$
\int_{0}^{T} e^{-\rho t} E\left[e^{-p U_{t}}\right] d t=\left\{\begin{array}{ll}
\frac{e^{T(\kappa(p)-\rho)}-1}{\kappa(p)-\rho} e^{-p u}, & \text { if } \kappa(p)-\rho \neq 0 . \\
T e^{-p u}, & \text { if } \kappa(p)-\rho=0 .
\end{array} .\right.
$$

Now, since we have

$$
0 \leq g(x) \leq K e^{-p x}
$$

for all $x \in \mathbb{R}$, it comes

$$
\int_{0}^{T} e^{-\rho t} E\left[g\left(U_{t}\right)\right] d t \leq K \int_{0}^{T} e^{-\rho t} E\left[e^{-p U_{t}}\right] d t
$$

Example 13 In the particular case where $g(x)=1_{\{x<0\}}$, we easily see that $K=1$ is the smallest positive constant satisfying (28), so that (29) holds true with $K=1$.

Example 14 If we consider $g(x)=|x|^{n} 1_{\{x<0\}}$ with $n=1,2, \ldots$, then we can show that $K=\left(\frac{n}{e p}\right)^{n}$ is the smallest positive constant satisfying (28), so that (29) holds true with $K=\left(\frac{n}{e p}\right)^{n}$. Indeed, the following equation

$$
|x|^{n}=K e^{-p x}, \quad x<0,
$$

admits the two solutions

$$
x=\frac{n}{p} W_{k}\left(\frac{-p K^{1 / n}}{n}\right), \quad k=0,-1,
$$

when $-p \frac{K^{1 / n}}{n}>-\frac{1}{e}$ where $W_{0}$ and $W_{-1}$ are the two branches of the Lambert function. Both solutions coincide when $K=\left(\frac{n}{e p}\right)^{n}$.

Specifically, Example 14 with $n=1$ and $\rho=0$ tells us that the expected area in red satisfies

$$
\mathcal{A}(u, T) \leq \begin{cases}\frac{e^{T \kappa(p)}-1}{e p \kappa(p)} e^{-p u}, & \text { if } \kappa(p) \neq 0 \\ \frac{T}{e p} e^{-p u}, & \text { if } \kappa(p)=0\end{cases}
$$

for all $u \geq 0$. This later inequality can be compared to the one deduced from Biard et al. (2010) when $u \rightarrow \infty$ in the compound Poisson process. Indeed, when there is a positive constant $p$ solution 
to the equation $\kappa(p)=0$ (called the adjustment coefficient), we know from Proposition 3.12 of Biard et al. (2010) that the expected area in red satisfies

$$
\mathcal{A}(u, T) \leq \mathcal{A}(u, \infty) \sim \frac{1}{c(1-\psi(0))} \frac{C}{p^{2}} e^{-p u} \quad \text { as } u \rightarrow \infty,
$$

where $\psi(0)$ is the infinite time ruin probability for $u=0$ and

$$
C=\frac{c-\lambda \mu}{\lambda \xi^{\prime}(p)-1}
$$

is the Cramer Lundberg constant (the notation is from Section 3.4). One sees that the upper bound (32) is smaller than the upper bound $(33)$ for $T<\frac{e C}{c(1-\psi(0)) p}$.

\section{$5 \quad$ Asymptotic behavior for subexponential claim distributions}

To end this paper, we assume that the Lévy measure $\nu$ satisfies $\bar{\nu}(x)=\int_{x}^{\infty} \nu(d y) \sim \bar{B}(x)$ as $x \rightarrow \infty$, where $\bar{B}(x)=1-B(x)$, for some distribution function $B$ such that the integrated tail $B_{0}(x)=\int_{x}^{\infty} \bar{B}(y) d y$ is subexponential, that is, we suppose that $B_{0}$ satisfies

$$
\bar{B}_{0}^{* 2}(x) \sim 2 \overline{B_{0}}(x) \text { as } x \rightarrow \infty,
$$

where $\bar{B}_{0}(x)=1-B_{0}(x)$ and

$$
B_{0}^{* 2}(x)=\int_{0}^{\infty} B_{0}(x-y) d B_{0}(y)
$$

Proposition 15 Suppose that $g$ is a continuously differentiable function on $(-\infty, 0)$. We have

$$
\mathcal{E}_{g}(u, T) \sim\left\{\begin{array}{ll}
\frac{1-(\rho T+1) e^{-\rho T}}{\rho^{2}} \int_{u}^{\infty} g(u-y) \nu(d y) & \text { for } \rho>0 \\
\frac{T^{2}}{2} \int_{u}^{\infty} g(u-y) \nu(d y) & \text { for } \rho=0
\end{array} \quad \text { as } u \rightarrow \infty\right.
$$

Proof. Lemma XI.2.2 in Asmussen and Albrecher (2010) gives that $\bar{F}_{X_{t}}(u) \sim t \bar{\nu}(u)$ as $u \rightarrow \infty$. Therefore, $\int_{u}^{\infty} g(u-y) d F_{X_{t}}(y) \sim t \int_{u}^{\infty} g(u-y) \nu(d y)$ as $u \rightarrow \infty$. Indeed,

$$
\left(f_{X_{t}} * g\right)(u)=-\left(\bar{F}_{X_{t}} * D g\right)(u) \sim-(t \bar{\nu} * D g)(u)=(-t D \bar{\nu} * g)(u)=t \int_{u}^{\infty} g(u-y) \nu(d y)
$$

as $u \rightarrow \infty$ since given $\epsilon>0$, we know that there exists $\rho>0$ such that, for all $y>\rho$, we have

$$
\left|\frac{\bar{F}_{X_{t}}(y)}{t \bar{\nu}(y)}-1\right| \leq \epsilon
$$


and so

$$
\left|\frac{\int_{u^{-}}^{\infty}\left(g^{\prime}(u-y)-g(u-y) \delta(y)\right) \bar{F}_{X_{t}}(y) d y}{\int_{u^{-}}^{\infty}\left(g^{\prime}(u-y)-g(u-y) \delta(y)\right) t \bar{\nu}(y) d y}-1\right|=\left|\frac{\int_{u^{-}}^{\infty}\left(g^{\prime}(u-y)-g(u-y) \delta(y)\right)\left(\frac{\bar{F}_{X_{t}}(y)}{t \bar{\nu}(y)}-1\right) t \bar{\nu}(y) d y}{\int_{u^{-}}^{\infty}\left(g^{\prime}(u-y)-g(u-y) \delta(y)\right) t \bar{\nu}(y) d y}\right| \leq \epsilon
$$

Hence, Proposition 1 yields

$$
\mathcal{E}_{g}(u, T) \sim \int_{0}^{T} e^{-\rho t} t\left(\int_{u}^{\infty} g(u-y) \nu(d y)\right) d t \quad \text { as } u \rightarrow \infty
$$

so that expression (34) directly follows.

A similar result to Proposition 15 can also be derived for $T=\infty$.

\section{Corollary 16}

$$
\mathcal{E}_{g}(u, \infty) \sim \frac{1}{\left|E\left[X_{1}\right]\right|^{2}}\left(\int^{\infty} \bar{\nu}(y) d y * g\right)(u)
$$

Proof. Theorem XI.2.1 in Asmussen and Albrecher (2010) directly leads to the approximation

$$
\psi(u) \sim \frac{1}{\left|E\left[X_{1}\right]\right|} \int_{u}^{\infty} \bar{\nu}(y) d y \quad \text { as } u \rightarrow \infty .
$$

Then, using Proposition 8 ends the proof.

In the compound Poisson model, Proposition 15 directly leads to the next result.

Corollary 17 If $F_{W}$ is an absolutely continuous distribution of the subexponential type, then we have

$$
\mathcal{E}_{g}(u, T) \sim\left(f^{(\infty)} * g\right)(u) \quad \text { as } u \rightarrow \infty,
$$

where

$$
f^{(\infty)}(v)=\left\{\begin{array}{ll}
\frac{1-(\rho T+1) e^{-\rho T}}{\rho^{2}} \lambda f_{W}(v) & \text { for } \rho>0 \\
\frac{\lambda T^{2}}{2} f_{W}(v) & \text { for } \rho=0
\end{array},\right.
$$

with $f_{W}$ being the density function of $W$.

Corollary 17 is an extension of

$$
\mathcal{A}(u, T) \sim \frac{\lambda T^{2}}{2} \int_{u}^{\infty} \bar{F}_{W}(x) d x \quad \text { as } u \rightarrow \infty
$$

derived in Biard et al. (2010) to any function $g$ and $\rho \geq 0$. Expression (37) is a particular case of (36) with $g(x)=|x| 1_{\{x<0\}}$.

Example 18 Let $\rho=0$ and $F_{W}(x)=\left(1-x^{-k}\right) 1_{\{x>1\}}$ with $k>1$ an integer, that is, $F_{W}$ is a 
Pareto distribution. Then, we get

$$
f^{(\infty)}(u) \sim \frac{\lambda T^{2}}{2} \frac{k}{u^{k+1}}, \quad \text { as } u \rightarrow \infty .
$$

Hence, for $g(x)=|x|^{n} 1_{\{x<0\}}$ with $0 \leq n<k$, (36) becomes

$$
\begin{aligned}
\mathcal{E}_{g}(u, T) & \sim \int_{-\infty}^{\infty} f^{(\infty)}(y) g(u-y) d y \\
& =\frac{k \lambda T^{2}}{2} \int_{u}^{\infty} \frac{(z-u)^{n}}{z^{k+1}} d z \\
& =\frac{k \lambda T^{2}}{2} \int_{u}^{\infty} \frac{\sum_{j=0}^{n}\left(\begin{array}{c}
n \\
j
\end{array}\right) z^{n-j}(-u)^{j}}{z^{k+1}} d z \\
& =\frac{k \lambda T^{2}}{2} \sum_{j}\left(\begin{array}{c}
n \\
j
\end{array}\right)(-u)^{j} \int_{u}^{\infty} z^{n-j-k-1} d z \\
& =\frac{k \lambda T^{2}}{2} \sum_{j} \frac{\left(\begin{array}{c}
n \\
j
\end{array}\right)(-u)^{j}}{n-j-k}\left(-u^{n-j-k}\right) \\
& =\frac{k \lambda T^{2}}{2} \frac{1}{u^{k-n}} \sum_{j=0}^{n} \frac{\left(\begin{array}{c}
n \\
j
\end{array}\right)(-1)^{j+1}}{n-j-k} \\
& =C \frac{1}{u^{k-n}}
\end{aligned}
$$

as $u \rightarrow \infty$, where

$$
C=\frac{k \lambda T^{2}}{2} \sum_{j=0}^{n} \frac{\left(\begin{array}{c}
n \\
j
\end{array}\right)(-1)^{j+1}}{n-j-k}
$$

\section{References}

1. Asmussen, S., Albrecher, H., 2010. Ruin Probabilities. World Scientific, Singapore.

2. Biard, R., Loisel, S., Macci, C., Veraverbeke, N., 2010. Asymptotic behavior of the finite-time expected time-integrated negative part of some risk processes and optimal reserve allocation. Journal of Mathematical Analysis and Applications 367, 535-549.

3. dos Reis, A. E., 1993. How long is the surplus below zero? Insurance: Mathematics and Economics 12, 23-38.

4. Embrechts, P., Goldie, C.M., Veraverbeke, N., 1979. Subexponentiality and infinite divisibility, Zeitschrift für Warhscheinlichkeitstheorie und Verwandte Gebiete 49(3), 335-347.

5. Gel'fand, I. M., Shilov, G. E., 1964. Generalized Functions, Volume 1: Properties and Operations, American Mathematical Soc.

6. Loisel, S., 2005. Differentiation of some functionals of risk processes, and optimal reserve allocation. Journal of Applied Probability 42, 379-392. 
7. Loisel, S., Trufin, J., 2014. Properties of a risk measure derived from the expected area in red. Insurance: Mathematics and Economics 55, 191-199.

8. Macci, C., 2008. Large deviations for the time-integrated negative parts of some processes. Statistics and probability letters $78(1), 75-83$.

9. Schwartz, L., 1945. Généralisation de la notion de fonction, de dérivation, de transformation de Fourier et applications mathématiques et physiques. Annales de l'Université de Grenoble $21,57-74$. 